\title{
Analysis of pressure-flow data in terms of computer-derived urethral resistance parameters
}

\author{
R. van Mastrigt and M. Kranse \\ Department of Urology-Urodynamics, Erasmus University Rotterdam, Rotterdam, The Netherlands
}

\begin{abstract}
Summary. The simultaneous measurement of detrusor pressure and flow rate during voiding is at present the only way to measure or grade infravesical obstruction objectively. Numerous methods have been introduced to analyze the resulting data. These methods differ in aim (measurement of urethral resistance and/or diagnosis of obstruction), method (manual versus computerized data processing), theory or model used, and resolution (continuously variable parameters or a limited number of classes, the so-called nomogram). In this paper, some aspects of these fundamental differences are discussed and illustrated. Subsequently, the properties and clinical performance of two computer-based methods for deriving continuous urethral resistance parameters are treated.
\end{abstract}

Consensus exists that infravesical obstruction can be diagnosed urodynamically only from simultaneous measurements of the detrusor pressure and flow rate during voiding, a pressure-flow study. In extreme cases the diagnosis is straightforward. If patients have a very high maximal flow rate and a very low detrusor pressure, they are obviously unobstructed. If they have a very low flow rate and a very high detrusor pressure the patients are obstructed [2]. In the great majority of cases, i.e., patients with moderate flow rates and/or moderate pressures, patients with low flow rates and/or low pressures, or patients with high flow rates and high pressures, such a simple, direct diagnosis is not possible. In such cases, methods of quantifying or grading the urethral resistance, or the degree of obstruction, of the patient can favorably be used.

Several different kinds of such methods have been developed. All these methods are based on pressure-flow data that are somehow manipulated to derive one or more parameters that represent urethral resistance and/or classify the pressure/flow data into one of a limited number of classes. The methods differ in a number of aspects: they are based on different models and/or on different dataprocessing techniques (automatic/computerized versus

Correspondence to: R. van Mastrigt, Department of Urology-urodynamics, room EE630, Erasmus University Rotterdam, P.O. Box 1738, NL-3000 DR Rotterdam, The Netherlands,

Fax $+(10) 4366428$ manual), have different aims (e.g., measurement of urethral resistance and/or diagnosis of obstruction), and have a different resolution, i.e., the smallest change in urethral resistance that can be detected is different.

In this article, some introductory remarks are made on the different models that are used, on urethral resistance factors, and on the required resolution for different aims. Subsequently, two methods for deriving urethral resistance factors and some examples of the clinical application of these methods are discussed in detail. Both of the methods imply computerized data processing. Finally, some statistics are applied to the criteria for selection of patients for pressure-flow studies.

\section{Models used for calculating urethral resistance parameters}

Most simply, the bladder outlet during voiding can be modeled as a hole in the urinary bladder [21]. In such an oversimplified case the relation between the detrusor pressure and the flow rate through the hole during voiding would be quadratic. The "classic" urethral resistance factor $\mathrm{p} / \mathrm{Q}^{2}[14]$ is based on this theoretical relation. Practical measurements, however, hardly ever show a simple quadratic curve. Figure 1 shows a typical example of a pressure-flow plot. The lowest pressure values at each flow rate represent the most relaxed state of the urethra and, thus, the best estimate for the degree of "anatomical" obstruction. Hereafter, these lowest values are referred to simply as the pressure flow data or the pressure-flow plot.

A simple quadratic curve is not applicable to the data shown in Fig. 1, as the curve does not pass through the origin [12]. This is the case because the urethra is not a hole in the bladder wall but a complicated structure that must be described as a flexible collapsible tube. A certain minimal pressure is needed to open this collapsed tube and keep it open during voiding. The terminology used for this minimal pressure is very confusing. Throughout this paper it will be called the theoretical urethral opening pressure, and in Fig. 1 it is in the order of $30 \mathrm{cmH}_{2} \mathrm{O}$. Taking into account a nonzero theoretical urethral opening pressure, a theoretical pressure-flow relationship can be derived as shown fitted to the data in Fig. 1. This has been called the passive urethral resistance relation (PURR) 


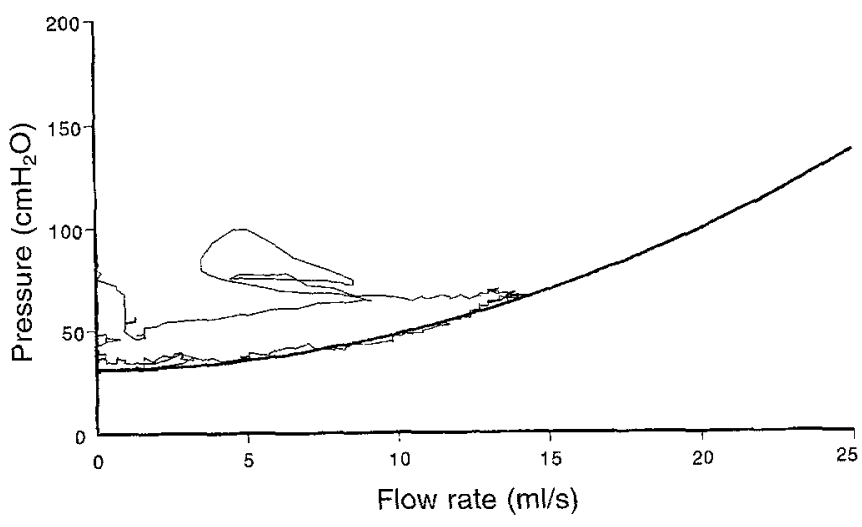

Fig. 1. A typical example of a pressure-flow plot, fitted with a quadratic pressure-flow relation with additive theoretical urethral opening pressure

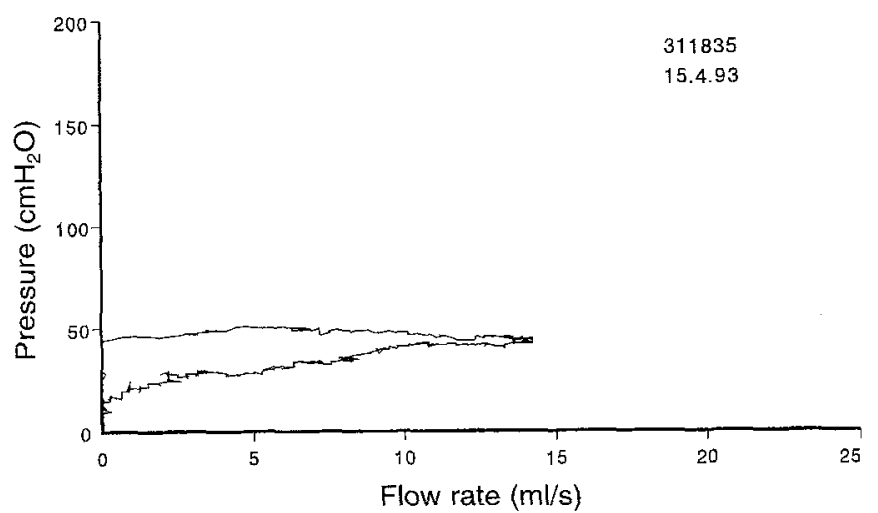

Fig.2. An example of a pressure-flow plot that cannot adequately be described by a quadratic pressure-flow relation

[20]. Although this relation accounts for opening of the urethra, it is nonetheless based on rigid pipe hydrodynamics: as long as the urethra is open, it has a constant crosssectional area. In reality this is usually not the case. The urethra is an elastic structure, and its cross-sectional area depends to some degree on the pressure. As a consequence, pressure-flow plots often have a shape that cannot adequately be described by the quadratic relation shown in Fig. 1. Figure 2 shows an example.

Taking a pressure-dependent change in the cross-sectional area of the urethra into account, it is possible to model the urethra as an elastic collapsible tube [3]. Such a model results in a pressure-flow relationship that is not quadratic but has a variable shape depending on the elasticity of the urethra [23]. In a subsequent section of this article we demonstrate that for the determination of one practical, combined urethral resistance parameter this model has drawbacks as a result of its statistical properties. Therefore, an alternative model with favorable statistical properties has also been proposed, an orthogonal polynomial model [7]. Using this model it was found that on average, pressure-flow plots can best be modeled in terms of the average height (of the lowest pressure values at each flow rate) and the average slope (of these values). Figure 3 shows a straight line based on these values superimposed on the data. This line does not ideally "fit" the

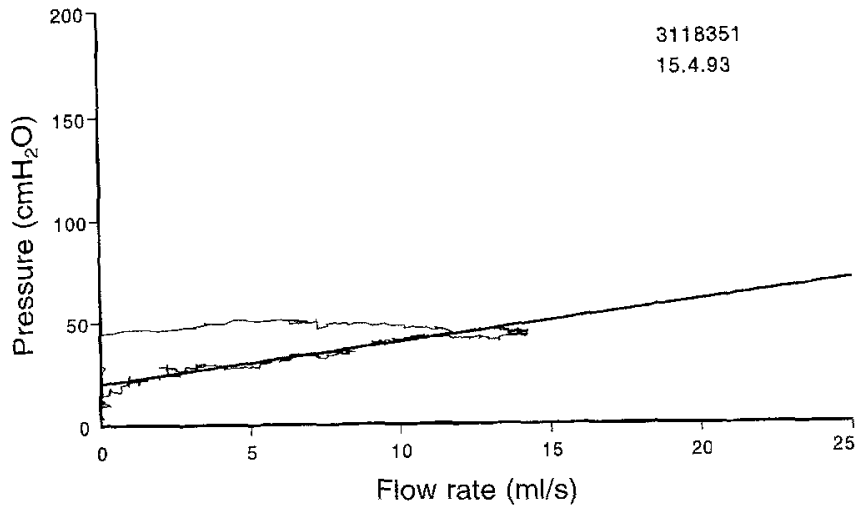

Fig. 3. A straight line fitted to the pressure-flow plot shown in Fig. 2. The line is defined by its average height and slope, which were automatically determined from the lowest pressure values in the data

data (as compared with other possible curves) but represents the only two properties (average slope and height) that can reliably be determined in the average patient.

\section{Urethral resistance parameters}

When the mathematical relations or models described in the previous section are used to characterize the pressureflow data measured in patients, a number of parameters result that define the fitted curve. The quadratic PURR curve (Fig. 1) is defined by two parameters: the theoretical urethral opening pressure, or the pressure at which the fitted curve intersects the pressure axis, and its steepness (in the references cited, only the theoretical urethral opening pressure is used as a parameter). The collapsible tube model [23] is defined by a comparable theoretical urethral opening pressure, a steepness, and a shape factor, or exponent $m$. The orthogonal polynomial model is defined by a number of parameters, depending on the number of terms included in the model; in Fig. 3, two terms were used, resulting in a curve defined by its average height and average slope.

In theory, the parameters listed for the various models can directly be used as urethral resistance parameters. A problem is that it is unclear how patient measurements should be compared and how, for instance, improvement or deterioration should be concluded if urethral resistance is defined in terms of a set of parameters. This can be illustrated using the study described below [13].

\section{Application of the orthogonal polynomial model to alpha-blocker data}

In 32 patients, 3 pressure-flow studies were done before treatment and 3 studies were conducted after 4 weeks of treatment; 16 patients were treated daily with $4 \mathrm{mg}$ of an alpha-blocker, doxazosin, and 16 patients received a placebo. The study was randomized and double-blind. The digitally stored pressure-flow data were processed using the software packages MATLAB, QPRO, PARADOX, 
Table 1. Mean value and standard deviation of the average height and the average slope of pressure-flow plots as measured in 16 patients treated daily with $4 \mathrm{mg}$ of Doxazosin and in 16 patients treated with placebo as well as the significance of the observed change in the parameters as a result of the treatment

\begin{tabular}{|c|c|c|c|c|c|c|}
\hline \multirow[t]{2}{*}{ Parameter } & \multirow[t]{2}{*}{ Treatment } & \multicolumn{2}{|c|}{$\begin{array}{l}\text { Before } \\
\text { treatment }\end{array}$} & \multicolumn{2}{|c|}{$\begin{array}{l}\text { During } \\
\text { treatment }\end{array}$} & \multirow{2}{*}{$\begin{array}{l}\text { Signifi- } \\
\text { cance of } \\
\text { change }^{a}\end{array}$} \\
\hline & & Mean & SD & Mean & SD & \\
\hline \multirow{2}{*}{$\begin{array}{l}\text { Average height } \\
\qquad\left(\mathrm{cmH}_{2} \mathrm{O}\right)\end{array}$} & Placebo & 66 & 26 & 63 & 29 & 0.72 \\
\hline & $4 \mathrm{mg}$ & 81 & 33 & 71 & 27 & 0.098 \\
\hline \multirow{2}{*}{$\begin{array}{l}\text { Average slope } \\
\qquad\left(\mathrm{cmH}_{2} \mathrm{O} / \mathrm{ml} / \mathrm{s}\right)\end{array}$} & Placebo & 4.5 & 4.6 & 4.6 & 3.7 & 0.57 \\
\hline & $4 \mathrm{mg}$ & 5.3 & 4.0 & 4.8 & 4.0 & 0.21 \\
\hline
\end{tabular}

a Wilcoxon matched-pairs signed-rank test and SPSS. The part of the presssure-flow data closest to the flow-rate axis was automatically detected and fitted with a first-order orthogonal polynomial [7]. The pressure-flow plots were thus characterized in terms of the two parameters average height and average slope. In Table 1 the mean value and standard deviation of these two aspects of the pressure-flow plots and the significance of the observed change in parametric values as a result of the treatment (Wilcoxon's matched-pairs signedrank test) are shown.

The table shows that both the average height and the average slope of the polynomial fitted to the pressureflow data decreased upon doxazosine treatment, but these changes were not significant. In the placebo group the average slope increased slightly but not significantly. The average height decreased insignificantly, and certainly to a lesser extent, in this group than in the treatment group. In terms of the significance values listed in the last column, the impression is gained that changes were more important in the treatment group than in the placebo group, but all differences were insignificant.

However, when average height and average slope were combined in a test variable by adding both values divided by the population means, the Wilcoxon matched-pairs signed-rank test showed a significant change in this test variable in the 4-mg group $(P=0.030)$ but no significant change in the placebo group $(P=0.76)$. This example therefore demonstrates that by combining different aspects or parameters of a pressure-flow plot into one urethral resistance factor or test variable, differences between patients treatments can be tested more sensitively.

\section{Combined urethral resistance parameters and nomograms}

Two fundamentally different methods are in use for combining aspects of pressure-flow data into one factor or class. One method is to use a nomogram or classification method that implies the use of borderlines or decision rules, for instance, "if the maximal flow rate is below soand-so much and the pressure is at least so-many times the flow rate but below so-many times the flow rate, the patient has obstruction type such-and-such" $[1,5,22]$. Another method is to calculate a continuous variable such as flow rate divided by pressure. Apart from obvious differences (for instance, some methods necessitate the use of a computer, whereas others do not), these two types of dif- ferent methods have partly different aims: a continuous variable is more fit for measuring urethral resistance and testing for changes, whereas a nomogram is more fit for diagnosing obstruction. The difference between these aims is nonsymmetrical. A continuous variable designed for measuring urethral resistance can be used for diagnosing obstruction (by adding borderlines or decision rules); a nomogram or classification method, however, often cannot be used for measuring urethral resistance. This results from the observation that a nomogram uses a limited number of discrete classes, in the extreme case only two: obstructed or unobstructed. This makes such methods less sensitive in detecting changes in outflow obstruction in response to treatment. Obviously, this must depend on the number of classes used in the nomogram. This dependence can be illustrated using the alpha-blocker data presented in the previous section.

To avoid bias by using specific pressure-flow analysis methods the detrusor pressure at maximal flow rate was considered as a measure of urethral resistance for this test. The (averaged) pressure values measured before and during treatment ranged up up $222 \mathrm{cmH}_{2} \mathrm{O}$. This range was divided into a varying number of equidistant classes. If a pressure value was identical to the borderline between two classes, a random generator was used to assign the value to one of the two neighboring classes. For each number of classes tested, the assignment procedure was repeated eight times to estimate the variation caused by the random assignment of borderline values. Figure 4 shows the significance of the difference observed between classified pressure values measured before and during treatment using the Wilcoxon matched-pairs signedrank test as a function of the number of equidistant classes.

The bar labeled $>200$ represents the significance of the difference for the original, unclassified pressure values (0.0231). The error bars represent the standard error of the mean as a result of repeated random assignment of borderline values. The arrows on top of the bars at five and four classes indicate that these bars are out of range. It can be observed that if the number of equidistant classes was less than or equal to nine, the difference in classified pressure values was not significant at a level of 0.05 . At three classes the significance seems to decrease again. This results from the observation that as the number of classes decreases, more and more patients are assigned to the same class before and during treatment. The statistical test used excludes these "ties." Figure 5 shows the 


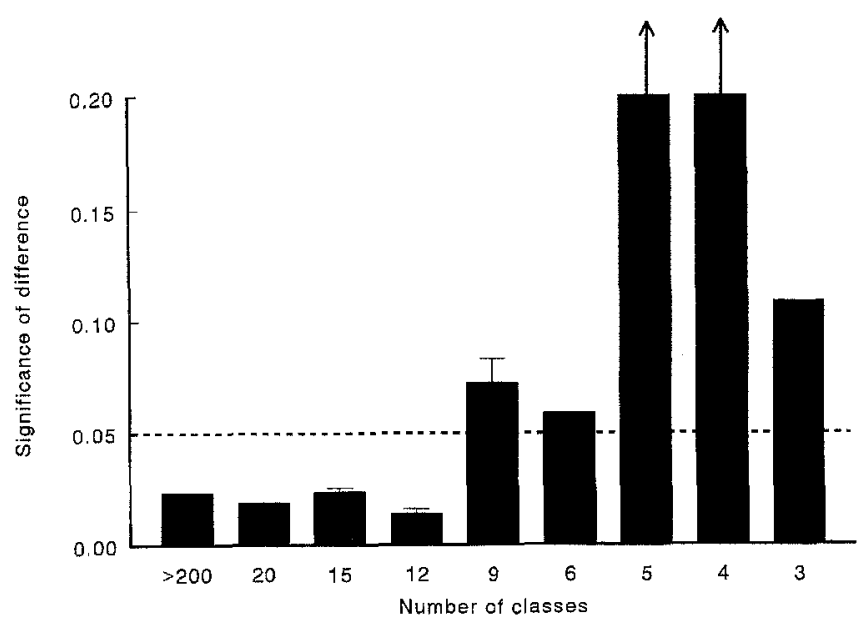

Fig.4. The significance of the observed decrease in detrusor pressure at maximal flow rate in patients treated with an alpha-blocker. The pressure values were classified using different numbers of equidistant classes. The bar labeled $>200$ gives the significance of the pressure change in the unclassified data. When 9 or fewer classes were used the pressure decrease was not significant at the $5 \%$ level

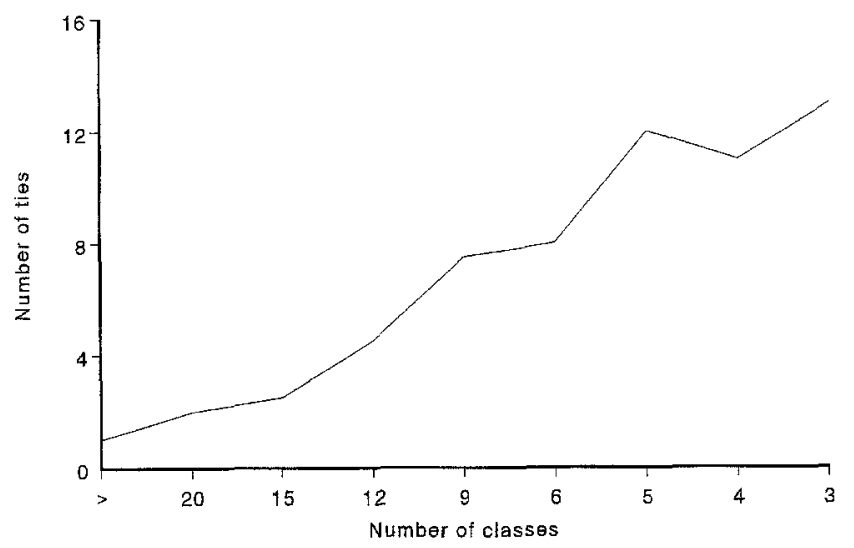

Fig. 5. The number of patients (of a total of 16) that were assigned to the same class before and during treatment with an alphablocker, plotted as a function of the number of equidistant classes used. Data were obtained from the patients illustrated in Fig.4

number of ties plotted as a function of the number of classes.

At 3 classes, 13 of the 16 patients were in the same class before and after treatment; in this case the significance level shown in Fig. 4 is based only on the remaining 3 patients. The graphs clearly illustrate that the classification of pressure-flow data into discrete classes may substantially limit the sensitivity for detecting changes in outflow resistance in response to treatment. In the tested population the significant change observed in detrusor pressure at maximal flow rate in response to treatment with an alpha-blocker was obscured if the data were classified using nine or fewer equidistant classes for one parameter. If several parameters are classified, the required number of classes increases. If all parameters behave similarly to the detrusor pressure at maximal flow rate, the required number of classes should be raised to the power of the number of parameters.

\section{Urethral resistance parameters URA and OBI}

From the models described above, only the simple quadratic model (without the theoretical urethral opening pressure) yields a single urethral resistance parameter, but as this model does not adequately describe clinical data, the resulting resistance factor $\mathrm{p} / \mathrm{Q}^{2}$ is unreliable. Two different approaches have been used to reduce or combine the sets of parameters that result from using the other three models into one urethral resistance parameter.

In the first appraoch the quadratic model (including the theoretical urethral opening pressure) illustrated in Fig. 1 was fitted to pressure-flow data of a mixed group of patients [4]. An experimental statistical relation was established between the two parameters of the model, theoretical urethral opening pressure and steepness. By inserting this relation into the model, an equation with only one parameter was derived. This one parameter has been called URA, and it can be calculated from any point along the pressure-flow curve. In all clinical applications the point of maximal flow rate and associated detrusor pressure is used.

In a second approach the parameters from the elastic collapsible tube model and the parameters from the orthogonal polynomial model shown in Fig. 3 were reduced to one urethral resistance parameter by using a statistical method called Fisher's linear discriminant. In this method, pressure-flow measurements are represented by a dot in an $n$-dimensional space ( $n$ being the number of parameters of the model) and the dots are projected on a line through the origin. The line is rotated until it best separates a group of obstructed and unobstructed patients. The distance along the line, then, is the new combined single obstruction parameter.

In the original publications $[23,24]$ on the application of the collapsible tube model, the authors used a discrete number of different values for the parameter describing the shape of the collapsible tube model. When Fisher's linear discriminant method was applied, a continuous version of the model was used, whereby the shape parameter could have any value. This resulted in very unreliable parameters, as one and the same pressure-flow plot could be fitted with several curves with completely different parameters. The orthogonal polynomial model did not suffer from this problem, as the parameters of this model are designed to be statistically independent. Combination of the parameters of the polynomial model using Fisher's linear discriminant method resulted in the single parameter OBI [8].

\section{Clinical applications of the parameters URA and OBI}

A few examples of the clinical application of the urethral resistance parameters URA and OBI are discussed below. With some exceptions, parametric values were calculated immediately following each urodynamic measurement using a special computer program. This program (CLIM [9, $10,16,19])$ enables the connection of a personal computer (PC) to urodynamic equipment and storage, retrieval, and analysis of urodynamic data. 
Table 2. The effect of surgical and pharmacological treatment of $\mathrm{BPH}$ patients on the average value $\pm \mathrm{SD}$ of the urethral resistance parameter URA and the significance according to the Wilcoxon signed-rank test

\begin{tabular}{lllll}
\hline $\begin{array}{l}\text { URA before } \\
\left(\mathrm{cmH}_{2} \mathrm{O}\right.\end{array}$ & Treatment & $\begin{array}{l}\text { URA after } \\
\left(\mathrm{cmH}_{2} \mathrm{O}\right)\end{array}$ & $\begin{array}{l}\text { Num } \\
\text { ber }\end{array}$ & Wilcoxon \\
\hline $40 \pm 21$ & Placebo & $39 \pm 23$ & 14 & $P=0.51$ \\
$51 \pm 20$ & TURP & $16 \pm 5$ & 19 & $P<0.0001$ \\
$53 \pm 21$ & 2 mg Doxazosin & $39 \pm 18$ & 16 & $P=0.03$ \\
$48 \pm 21$ & 4 mg Doxazosin & $41 \pm 18$ & 15 & $P=0.03$ \\
\hline
\end{tabular}

Table 3. The effect of surgical and pharmacological treatment of $\mathrm{BPH}$ patients on the average value $\pm \mathrm{SD}$ of the urethral resistance parameter $\mathrm{OBI}$ and the significance according to the Wilcoxon signed-rank test

\begin{tabular}{lllll}
\hline $\begin{array}{l}\text { OBI } \\
\text { before }\end{array}$ & Treatment & OBI after & $\begin{array}{l}\text { Num- Wilcoxon } \\
\text { ber }\end{array}$ \\
\hline $68 \pm 23$ & Placebo & $62 \pm 27$ & 14 & $P=0.30$ \\
$83 \pm 34$ & TURP & $34 \pm 10$ & 19 & $P=0.0001$ \\
$88 \pm 31$ & 2 mg Doxazosin & $71 \pm 28$ & 16 & $P=0.16$ \\
$94 \pm 35$ & 4 mg Doxazosin & $80 \pm 30$ & 15 & $P=0.04$ \\
\hline
\end{tabular}

In a small pilot study of ten patients [6], the diameter of the prostatic urethra was measured from transrectal ultrasound recordings at the apex, the midurethra, and the bladder neck. Measurements were taken at maximal flow rate. Spearman's rank correlations between the three diameters and URA were $-0.70(P=0.013),-0.48(P=$ $0.079)$, and $-0.71(P=0.011)$, respectively. Rank correlations with OBI were $-0.62(P 0.027),-0.60(P=0.032)$, and $-0.60(P=0.032)$, respectively. This finding shows that the urethral resistance as quantified by the parameters URA and OBI is determined to a large degree by the urethral diameter during voiding.

URA and OBI were also determined in a series of 29 patients before and after transurethral resection of the prostate (TURP) $[11,15,17]$. These patients were selected using conventional clinical criteria. It was found that the population consisted of two groups: a group of obstructed patients with a high URA value before the operation and a significantly decreased URA value afterward $(n=19)$ and a smaller but significant group $(n=10$, approximately $35 \%$ ) of unobstructed patients with low URA values both before and after the operation. The outcome of surgery in terms of subjective symptomatology was less favorable in these patients, which provides strong arguments for including preoperative testing with the described parameters before prostatic surgery [17].

Table 2 shows the change in URA observed in response to TURP in obstructed patients as compared with that seen in response to various doses of the alpha-blocker Doxazosine [18]. The table shows that although the effect of this drug is dramatically smaller than that of the operation, there was nevertheless a significant reduction in the parameter URA in the group of patients treated with $2 \mathrm{mg}$ as well as in that treated with $4 \mathrm{mg}$. Table 3 shows similar data for the urethral resistance parameter OBI in the same groups. Apart from the group treated with TURP, this parameter shows a significant change only in the 4-mg group.

\section{Patient selection for pressure-flow analysis}

The analysis methods and urethral resistance parameters discussed offer a promising tool for diagnosis and treatment of lower-urinary-tract disorders. All methods are based on an invasive and costly measurement of detrusor pressure in relation to flow rate. Many attempts have been made to quantify or define obstruction using a cheap, easy, and noninvasive flow-rate measurement alone. It can easily be shown that this is fundamentally impossible. On the other hand, it is very unlikely that patients with an extremely high maximal flow rate are obstructed, and it is probably equally unlikely that patients with a very low flow rate are unobstructed. Given that in a practical situation, not even a pressure-flow study can give $100 \%$ certainty that a patient is obstructed or unobstructed (after all, there is not a $100 \%$ reproducibility of studies), it must be possible to design a strategy that limits the number of pressure-flow studies without significantly affecting the diagnostic accuracy.

A first attempt at such a strategy was developed using a total of 109 pressure-flow studies of men consecutively evaluated in our outpatient clinic. Only studies with unimodal flow-rate curves and pressure-flow studies without artefacts were included. In case repeated studies were available, the study with the highest maximal flow rate was chosen for analysis. The patients were diagnosed as obstructed or unobstructed using the urethral resistance parameter URA and a cutoff value of $29 \mathrm{cmH}_{2} \mathrm{O}$. Assuming the diagnosis based on the pressure-flow analysis to be $100 \%$ correct, the population consisted of 70 unobstructed and 39 obstructed patients. Discriminant analysis showed that with the use of flow-rate measurements alone, these groups of patients could best be separated with a cutoff value of $10.4 \mathrm{ml} / \mathrm{s}$ (one patient in this population voided $120 \mathrm{ml}$ and all other patients voided $150 \mathrm{ml}$ or more, implying that the volume dependence of the maximal flow rate did not bias the data). Basing the diagnosis of all patients on flow rate alone yielded a diagnostic accuracy of $77 \%$, assuming that the diagnosis based on the pressure-flow study was $100 \%$ accurate. This figure should be related to the diagnostic accuracy that can be attained by blindly diagnosing all patients in this population as unobstructed. This diagnosis is correct in $70 / 109$ or $64 \%$ of cases. Thus, in this population the use of flow rate as a diagnostic tool for detecting obstruction increased the diagnostic accuracy by $13 \%$. Figure 6 shows the diagnostic accuracy plotted as a function of the percentage of patients in whom pressure-flow studies are done.

The figure shows that doing only pressure-flow studies in the $62 \%$ of patients with the lowest flow rates and relying on free flow-rate measurement in the other patients in this population results in a diagnostic accuracy of $100 \%$. A similar graph was constructed for the exclusion of patients with the lowest flow rates from pressure-flow analysis. This was a mirror image of Fig. 6 and led to the 


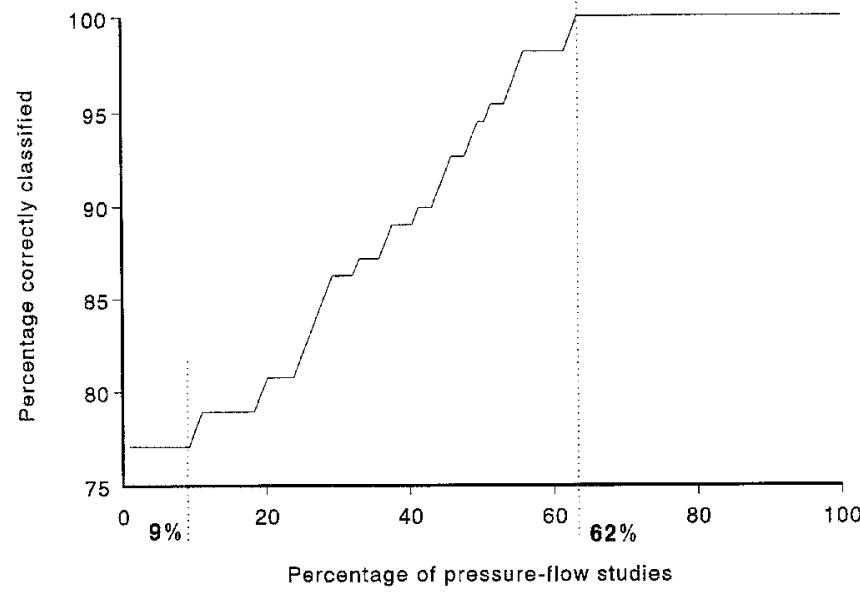

Fig. 6. The performance of a strategy in which only a limited percentage of the patients is subjected to pressure-flow analysis. This limited group of patients is preselected using free flow-rate measurements. The diagnostic accuracy of such a strategy is plotted as a function of the percentage of patients in which pressure-flow analysis is done, assuming that pressure-flow analysis is $100 \%$ accurate

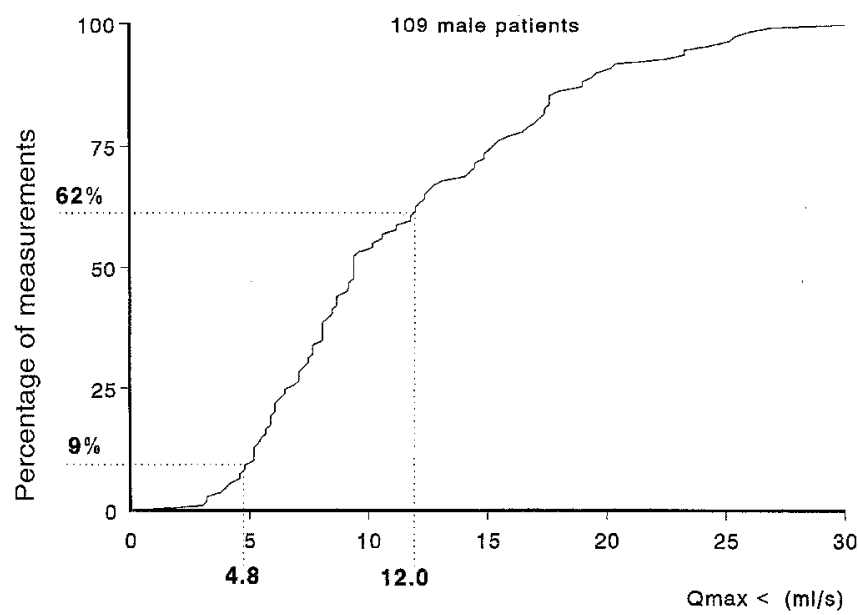

Fig.7. Cumulative-probability density function of the maximal flow rates measured in the patient group for which Fig. 6 was calculated. The graph shows the percentage of patients who had a maximal flow rate below a certain value

conclusion that the $9 \%$ of patients with the lowest flow rate did not need pressure-flow studies. The combination of these two exclusion strategies revealed that in the tested population, pressure-flow studies were necessary in only $62 \%-9 \%=53 \%$ of patients to yield an uncompromised diagnosis. The flow-rate cutoff values related to these percentages of the patient population can be read from Fig. 7, which illustrates the cumulative-probability density function of the maximal flow rate. It shows the percentage of patients in the tested population that had a flow rate lower than a certain value.

From this figure we can directly read that the $62 \%$ of patients that did not need a pressure-flow study according to Fig. 6 had a flow rate exceeding $12 \mathrm{ml} / \mathrm{s}$ and that the other $9 \%$ that did not need it had a flow-rate below 4.8 $\mathrm{ml} / \mathrm{s}$. The described data thus illustrate that it is possible to design a strategy to limit the number of pressure-flow studies in a given patient population by first investigating patients with a free flow-rate measurement and then doing pressure-flow studies only in those patients with a free flow rate ranging between certain flow-rate values (in this study, between 4.8 and $12 \mathrm{ml} / \mathrm{s}$ ). By the latter strategy, invasive measurement is necessary in only $53 \%$ of the patients in the tested population.

\section{Conclusions}

At present, a pressure-flow study is the only reliable method to measure or diagnose the degree of infravesical obstruction. Many methods have been proposed to analyze the resulting pressure-flow plot. These methods differ in aim, models used, data-processing techniques applied, and resulting resolution. Two aims can be distinguished: diagnosis of obstruction and measurement of urethral resistance (e.g., to evaluate the efficacy of treatment). Methods that aim at measuring urethral resistance can simply be extended with decision rules for diagnosing obstruction, whereas methods aiming at diagnosis often cannot be used for measuring urethral resistance; hence, the former may be called more universal.

From the available models, only the collapsible tube model [23] describes all measured patient data adequately. However, this model has statistical drawbacks. In practice, only the first two terms of an orthogonal polynomial that overcomes these problems can reliably be determined, implying that on average a simple straight line is the most adequate description of pressure-flow data. The independent parameters that best define this straight line (average height and average slope) can directly be used as urethral resistance parameters, but for simple diagnosis and sensitive testing of the efficacy of treatment it is advantageous to combine these parameters into one urethral resistance factor.

Some successful clinical applications of such a combined parameter (OBI) and an earlier single resistance factor (URA) have been discussed. Clinically, both perform comparably, but as URA is based on a less adequate model (quadratic with additive theoretical urethral opening pressure), it sometimes considerably "misfits" the pressure-flow data. Both of the parameters were calculated from the pressure-flow data using a computer (for URA this is not necessary, whereas for OBI it is). Apart from being efficient and enabling the use of more advanced dataanalysis methods, the application of a computer in analyzing urodynamic data ensures objectivity: all data are processed uniformly according to predefined rules, and no human observer error or bias is introduced. Such objectivity is required in statistical processing of data, for instance, to test the effect of pharmacological treatment. Of course, adequate methods should be implemented to avoid errors and bias caused by artefacts in the measurements.

Methods for analyzing pressure-flow data that do not involve computers (although all new urodynamic equipment systems available include a computer) are usually based on a classification system with a limited number of classes. Such methods have too low a sensitivity for test- 
ing the efficacy of treatment. The high cost and invasiveness of pressure-flow studies for diagnosing obstruction can be controlled by subjecting only patients who fall within a certain range of free flow rates to these studies. By carefully determining this range for each patient population and urodynamics center, it is possible to limit the number of necessary pressure-flow studies without affecting the diagnostic accuracy.

\section{References}

1. Abrams PH, Griffiths DJ (1979) The assessment of prostatic obstruction from urodynamic measurements and from residual urine. Br J Urol 51:129-134

2. Abrams P, Blaivas JG, Stanton SL, Andersen JT (1988) The standardisation of terminology of lower urinary tract function. Scand J Urol Nephrol [Suppl] 114:5-19

3. Griffiths DJ (1980) Urodynamics. The mechanics and hydrodynamics of the lower urinary tract. (Medical physics handbooks series, vol 4) Adam Hilger, Bristol

4. Griffiths D, Mastrigt R van, Bosch R (1989) Quantification of urethral resistance and bladder function during voiding, with special reference to the effects of prostate size reduction on urethral obstruction due to BPH. Neurourol Urodyn 8:17-27

5. Höfner K, Kramer AEJL, Tan HK, Grünewald V, Jonas U (1993) Chess classification of outflow obstruction based on pressure-flow analysis. Neurourol Urodyn 12:414-415

6. Jansen H, Mastrigt R van (1992) Transrectal ultrasound of the prostatic urethra related to urodynamically assessed urethral resistance. Neurourol Urodyn 11:401-403

7. Kranse M, Mastrigt R van (1991) Fitting orthogonal polynomials to the lowest part of a pressure flow plot. Neurourol Urodyn $\cdot 10: 290-291$

8. Kranse M, Mastrigt $\mathrm{R}$ van (1991) The derivation of an obstruction index from a three parameter model fitted to the lowest part of the pressure flow plot. J Urol 145:261A

9. Mastrigt R van (1984) A computer program for on-line measurement, storage, analysis and retrieval of urodynamic data. Comp Prog Biomed 18:109-117

10. Mastrigt $R$ van (1987) Urodynamic analysis using an one-line computer. Neurourol Urodyn 6:206-207
11. Mastrigt R van, Rollema HJ (1992) Prognostic value of bladder contractility in prostatectomy. J Urol 148:1856-1860

12. Mastrigt R van, Kranse M (1993) Automated evaluation of urethral obstruction. Urology 42:216-224

13. Mastrigt R van, Kranse M, Rollema HJ (1994) Testing the efficacy of treatment for infravesical obstruction with pressure flow data. Neurourol Urodyn 13:392-394

14. Pierce JM, Braun E, Sniderman AJ, Lewis HY (1963) The concept of resistance applied to the lower urinary tract. Surg Gynecol Obstet 116:217-222

15. Rollema HJ, Mastrigt R van (1987) Detrusor contractility before and after prostatectomy. Neurourol Urodyn 6:220-221

16. Rollema HJ, Mastrigt R van (1991) Objective analysis of prostatism; a clinical application of the computer program CLIM. Neurourol Urodyn 10:71-76 [Erratum in Neurourol Urodyn 10:267 (1991)]

17. Rollema HJ, Mastrigt $R$ van (1992) Improved indication and follow-up in transurethral resection of the prostate (TUR) using the computer program CLIM. J Urol 148:111-116

18. Rollema HJ, Rosier P, Janknegt RA, Mastrigt R van (1991) Efficacy of alpha-blocker (doxazosin) in BPH appraised by pressure-flow (CLIM) analysis. Neurourol Urodyn 10:295-296

19. Rollema HJ, Mastrigt R van, Janknegt RA (1991) Urodynamic assessment and quantification of prostatic obstruction before and after transurethral resection of the prostate; standardization with the aid of the computer program CLIM. Urol Int 47 [Suppl 1]:52-54

20. Schäfer W (1983) The contribution of the bladder outlet to the relation between pressure and flow rate during micturition. In: Hinman F Jr (ed) Benign prostatic hypertrophy. Springer, New York Berlin Heidelberg, pp 470-498

21. Schäfer W (1985) Urethral resistance? Urodynamic concepts of physiological and pathological bladder outlet function during voiding. Neurourol Urodyn 4:161-201

22. Schäfer W, Waterbär F, Langen PH, Deutz FJ (1989) A simplified graphic procedure for detailed analysis of detrusor and outlet function during voiding. Neurourol Urodyn 8:405-407

23. Spangberg A, Teriö H, Engberg A, Ask P (1989) Quantification of urethral function based on Griffiths' model of flow through elastic tubes. Neuroural Urodyn. 8:29-52

24. Teriö H, Spangberg A, Engberg A, Ask P (1989) Estimation of elastic properties in the urethral flow controlling zone by signal analysis of urodynamic pressure/flow data. Med Biol Eng Comput $27: 314-321$ 